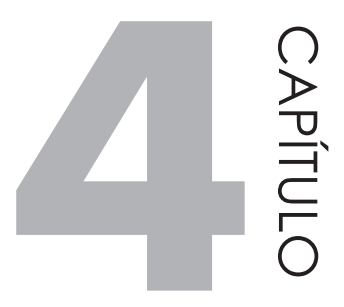

\title{
LEIS DE NEWTON
}

\subsection{PRIMEIRA LEI}

Galileu determinou, por intermédio de suas experiências, que uma esfera, em uma superfície plana, uma vez posta em movimento, continuaria indefinidamente, desde que as forças de atrito fossem eliminadas. Com base nisso, Newton formulou sua primeira lei.

A Primeira Lei, ou Lei da inércia, postula que qualquer corpo permanece em repouso ou em movimento retilíneo uniforme, a menos que seja obrigado a sair desse estado por forças aplicadas a ele.

Ou seja, se nenhuma força resultante atuar sobre um corpo sua aceleração $a$ será nula.

\subsection{SEGUNDA LEI: MASSA}

Chegamos ao conceito de massa como inércia, isto é, a resistência ao movimento: $a=\frac{F}{m}$

Quanto maior a massa, maior a força para a mesma aceleração. ${ }^{1}$

Assim, temos a equação fundamental da mecânica clássica: $\vec{F}=m \vec{a}$

[Ver observação da Seção 4.4, Equação (1), e da Seção 4.3, "Quantidade de movimento"].

A primeira lei está implícita na $2^{a}$ Lei, isto é, quando a força for nula, a aceleração também será nula.

\footnotetext{
Agora consideramos a relação entre força e aceleração, que é objeto da Dinâmica.
} 
44

Antonio Giuseppe Roth

4.3 QUANTIDADE DE MOVIMENTO

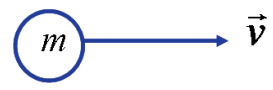

Seja a massa $m$ se deslocando com velocidade $\vec{v}$, chamamos quantidade de movimento ao produto de $m$ por $\vec{v}$.

$$
\vec{p}=m \vec{v}
$$

Originalmente, Newton descreveu a $2^{\mathrm{a}}$ Lei utilizando o conceito da quantidade de movimento [ver Anexo 9, Equação (2) sob título $2^{\mathrm{a}}$ Lei e Gravitação Universal].

Quando uma força age sobre um corpo, esta provoca a variação da sua quantidade de movimento em relação ao tempo, na direção da força:

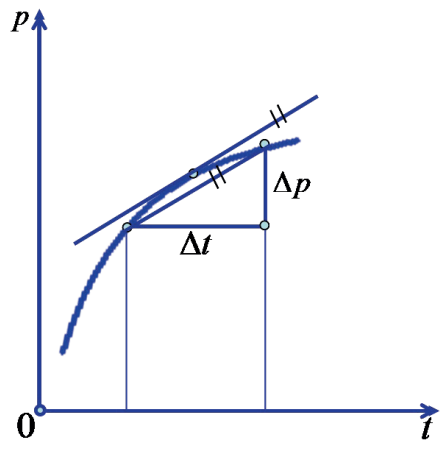

$$
\vec{F}=\lim _{\Delta t \rightarrow 0} \frac{\Delta \vec{p}}{\Delta t}=\frac{d \vec{p}}{d t}=\frac{d}{d t}(m \vec{v})
$$

Se a massa for constante: $\vec{F}=m \frac{d \vec{v}}{d t}$

mas a aceleração: $\vec{a}=\frac{d \vec{v}}{d t}$

Logo: $\vec{F}=m \vec{a}$ 


\subsubsection{Exemplo ilustrativo}

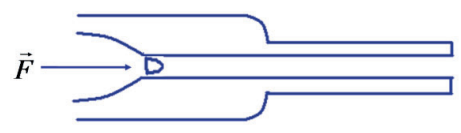

Um projétil impelido pelos gases da pólvora (que fornece a força) está sujeito à aceleração $\vec{a}$ (GAMOW; HALLIDAY, RESNICK).

\subsection{PESO E MASSA}

O peso de um corpo é a força gravitacional exercida sobre ele pela Terra. Exerce-se do corpo para o centro da Terra.

Nesse caso, a aceleração da gravidade $\vec{g}=\vec{a}$ e o peso $\vec{W}=\vec{F}$

$$
\text { Logo: } \vec{W}=m \vec{g}
$$

\subsubsection{Exemplo ilustrativo}

Se uma pessoa pesa $50 \mathrm{~kg} f$ na superfície da Terra, quanto pesará na superfície da Lua?

Sabendo que a relação das acelerações gravitacionais entre a Lua e a Terra é: $g_{\ell} / g_{t}=0,166$, o peso na superfície da Lua será: $50 \times 0,166=8,3 \mathrm{kgf}$ (HALLIDAY, RESNICK; ENC. BRITANNICA).

2 Rigorosamente, deveríamos expressar a $2^{a}$ Lei na forma: $F=k m a$, onde $k$ é uma constante de proporcionalidade que depende do sistema de unidades utilizado. Assim, no sistema MKSC, usamos massa $m$ em quilograma massa, $\mathrm{kgm}$, obtendo a força $F$ em Newtons, $N$, e $k$ sendo $k=1$, como mencionado na Seção 4.2.

No sistema MKS técnico, apresentado no fim da Seção 2.1, utilizamos também a massa $m$ em $k g m$, mas a força em quilograma força $k g f$, resultará em $k=\frac{\mathrm{kgf}}{\mathrm{kgm}} \times \frac{\mathrm{seg}^{2}}{\mathrm{~m}}$, diferente de 1: $k=1 / 9,81 \mathrm{kgf} \cdot \mathrm{seg}^{2} / \mathrm{kgm}^{\mathrm{m}}$.

De modo semelhante, no sistema gaussiano cgs, usamos a massa $m$ em grama $g$, obtendo a força em dinas, que resultará em $k=1$, e a fórmula resulta em $F=m a$, a expressão preferida pelos físicos.

Vale observar que no sistema MKS técnico, o valor da força em $\mathrm{kg} f$ é igual ao valor da massa em $\mathrm{kgm}$, considerando o valor da aceleração da gravidade $g=9,81 \mathrm{~m} / \mathrm{s}^{2}$ ao nível do mar, e $40^{\circ}$ de latitude, valor padrão, pois $g$ varia com a altitude e a latitude. 


\subsection{TERCEIRA LEI: AÇÃO E REAÇÃo}

A toda ação corresponde uma reação igual e de sentido contrário.

Se o corpo A exercer uma força sobre o corpo $B$, este exercerá sobre $A$ uma força igual, mas de sentido oposto. Nota-se que as forças de ação e reação atuam sobre corpos diferentes.

\subsubsection{Exemplo ilustrativo}

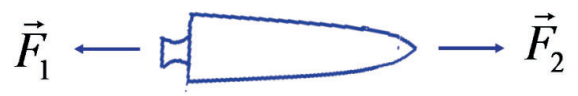

Os gases resultantes da queima do combustível do foguete se deslocam para trás através de um ejetor com alta velocidade. Como resultado, o corpo do foguete é empurrado para frente (HALLIDAYRESNICK; GAMOW).

\subsection{INTERAÇÃO GRAVITACIONAL}

Em 1665, Isaac Newton, então com 23 anos, mostrou que a gravitação que mantém a Lua em sua órbita é a mesma que faz uma maçã cair do galho de uma árvore. ${ }^{3}$ Ele concluiu que a Terra atrai tanto a maçã quanto a Lua, e também que: "Qualquer corpo no Universo atrai todos os outros”.

A partir disso, estabeleceu a Lei da Gravitação, que postula: a força gravitacional é diretamente proporcional às massas e inversamente proporcional ao quadrado da distância entre as massas.

$$
F=G \frac{m_{1} m_{2}}{r^{2}}
$$

$G$ é a constante de gravitação universal. Ver anexo 9, como Newton a deduziu.

$$
G=6,67 \times 10^{-11} N \cdot \mathrm{m}^{2} / \mathrm{kg}^{2}
$$

\section{Observação}

A ideia intuitiva de Newton é que as massas se atraem à distância, dando origem à fórmula da gravitação, semelhante à fórmula de cargas elétricas de sinais opostos que se atraem. Contudo, como será visto no segundo volume desta obra, a ideia de Einstein é que a massa deforma o espaço-tempo quadridimensional, fazendo que as massas em movimento próximas encurvem suas trajetórias.

Assim como a água descendo pela pia, como se o orifício atraísse essa água, isto é, imagine um objeto boiando na água e descendo pela pia, o objeto seria a massa atraída pelo orifício. Na verdade, o orifício

Publicada somente em 1687, intitulada Philosophiae Naturalis Principia Mathematica. Para uma compreensão de como Newton chegou à Lei da gravitação, ver Anexo 9. 
seria uma singularidade matemática nessa analogia, não algo que atraísse a matéria, como imaginava Newton. Ou melhor, imagine um espaço bidimensional representado por uma folha elástica quadriculada, onde se deposita uma bola. A bola provoca uma depressão e as linhas inicialmente retas na proximidade da bola agora se tornam curvas. Se lançarmos uma bolinha de gude em torno da depressão obtemos uma trajetória semelhante à de um planeta em torno do Sol. Nessa interpretação, devida a Einstein, a gravidade é uma deformação na estrutura do espaço bidimensional.

Imagine-se que vivemos em um Universo tridimensional, distorcido localmente pela matéria em uma $4^{a}$ dimensão física, a qual não pode ser percebida diretamente. Quanto maior a massa local, maior a gravidade local e maior a distorção, até se obter, em casos extremos, um buraco sem fundo, também conhecido como buraco negro (SAGAN; WILL; EINSTEIN).

A Lei dos períodos de Kepler postula que o quadrado do período de qualquer planeta é proporcional ao cubo do semieixo maior de sua órbita [ver Anexo 9, Equação (10), dedução da $3^{a}$ lei de Kepler].

Semieixo maior da elipse $a$.

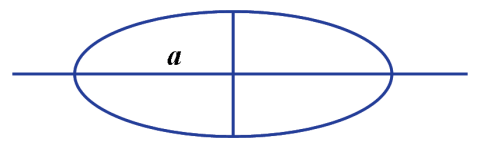

Consideremos uma órbita circular de raio $r$ (o raio é equivalente ao semieixo maior).

Aplicando $F=m a, \frac{G M}{r^{2}} m=(m) \cdot\left(\omega^{2} r\right) \quad$ e $\quad g=a$

$\omega^{2} r$ é a aceleração centrípeta

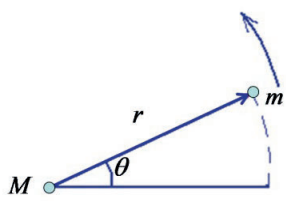

Substituímos $\omega=2 \pi / T$ em que $T$ é o período [ver Seção 2.1, "Movimento harmônico simples", Equação (3c)].

Ver na Equação (3), Seção 4.7, uma forma mais exata sugerida por Newton.

Encontramos: $T^{2}=\left(\frac{4 \pi^{2}}{G M}\right) r^{3}$ 
$\frac{4 \pi^{2}}{G M}$ é uma constante que depende só da massa do corpo central. Obtemos a lei dos períodos de Kepler. ${ }^{4}$

A equação vale também para órbitas elípticas, substituindo $r$ por $a$ [semieixo maior da elipse, a é proporcional a r, ver Dedução da $3^{a}$ Lei de Kepler no Anexo 9, Equação (10)]..$^{5}$

$$
T^{2} / a^{3}
$$

é a mesma para todas as órbitas planetárias (HALLIDAY, RESNICK, WALKER; ALONSO, FINN). $T^{2} / a^{3} \cong 3,0 \times 10^{-34} t^{2} / L^{3}$ sendo t em anos e $\mathrm{L} \mathrm{em} \mathrm{m},{ }^{6}$ mais exatamente, devemos usar a massa reduzida para todas as órbitas planetárias. A propósito, convém consultar a Lei de Titius-Bode, no final do Anexo 9.

Newton testou a validez comparando a aceleração centrípeta da Lua $a_{C} \operatorname{com}$ a aceleração da gravidade $^{7}$

$$
\begin{gathered}
g=9,80 \mathrm{~m} / \mathrm{s}^{2} \\
a_{c}=v^{2} / r=4 \pi^{2} r / T^{2}=\omega^{2} \mathrm{r}
\end{gathered}
$$

Ver Capítulo 2, Equação (3a1).

$$
\begin{aligned}
& r=3,84 \times 10^{8} \mathrm{~m}=384000 \mathrm{~km} \text { e } \quad \omega=\frac{2 \pi}{T} \quad T=2,36 \times 10^{6} \mathrm{~s}=27 \mathrm{~d} 7 \mathrm{~h} 33 \mathrm{~min} \cong 28 d \\
& a_{c}=2,72 \times 10^{-3} \mathrm{~m} / \mathrm{s}^{2} \\
& \text { Logo: }{ }^{8} \frac{g}{a_{c}}=3602 \cong(60)^{2}
\end{aligned}
$$

Raio da Terra: $R=6,37 \times 10^{6} \mathrm{~m}=6370 \mathrm{~km}$

Temos: $\left(\frac{r}{R}\right)^{2}=\left(\frac{384}{6,37}\right)^{2} \cong(60)^{2}$

O quadrado do período é proporcional ao cubo do semieixo maior, obtida experimentalmente por observação astronômica.

5 A fórmula $P^{2}=\frac{4 \pi^{2}}{\gamma m^{\prime}} a^{3}$, é mais geral, por levar em conta a área da elipse. A fórmula: $P^{2}=\frac{4 \pi^{2}}{G M} r^{3}$, adapta-se melhor quando a órbita é praticamente circular como a da Terra e a da Lua.

${ }^{6}$ Semieixo maior: $a$ em $10^{10} \mathrm{~m}$. Exemplo: para a Terra, $a=15$, isto é, $15 \times 10^{10} \mathrm{~m}=150.000 .000 \mathrm{~km}$ (HALLIDAY, RESNICK, WALKER).

A ideia é que a gravitação terrestre é compensada pela aceleração centrifuga, e a Lua continua em órbita por inércia.

8 Recordemos: $g=\frac{F}{m}=-\frac{G M}{r^{2}} \therefore$ A gravitação ocasionada pela massa da Terra $M$ é inversamente proporcional ao quadrado da distância entre o centro da Terra e o objeto atraído de massa $m$. 
Logo: $g / a_{c}=(r / R)^{2}$ (2) dentro da precisão dos cálculos, as duas acelerações estão na proporção inversa dos quadrados das distâncias dos pontos ao centro da Terra. Não confundir com a gravidade da Lua, que é devida à sua massa, como já visto, 0,166 vezes menor (ALONSO, FINN).

\subsubsection{Por que a força gravitacional entre duas massas é proporcional ao quadrado da distância que os separa?}

Com a apresentação da força gravitacional, deparamos com uma dúvida recorrente, conforme expresso no título desta subseção. Com base no que foi visto anteriormente, vemos como Newton justificou simplificadamente a dependência entre massa e sua distância (uma prova rigorosa de Newton será fornecida no Anexo 9): assim como a maçã que cai do galho de uma árvore é atraída pela Terra, a Lua também é atraída pelo planeta, pensou ele.

Como a órbita da Lua é aproximadamente circular, e o círculo é um caso particular da elipse, Newton aplicou a $1^{a}$ Lei de Kepler, que postula que a órbita dos planetas é elíptica. Nesse caso, os dois focos coincidem com o centro. A força F, centrípeta, aponta para o centro do círculo.

Aplicando a fórmula da força centrípeta em um movimento circular:

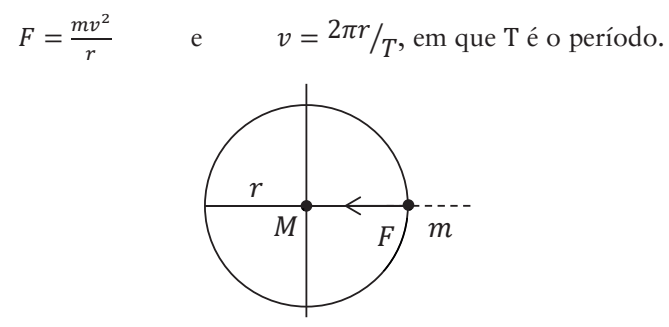

Pela $3^{\mathrm{a}}$ Lei de Kepler, para a órbita circular, temos que: o quadrado do período T é proporcional ao cubo do raio $\mathrm{r}: \mathrm{T}^{2}=\mathrm{kr}^{3}$.

$$
\text { Portanto, } F=\frac{m}{r}\left(\frac{2 \pi r}{T}\right)^{2}=\frac{m}{r} \frac{4 \pi^{2} r^{2}}{k r^{3}} \Rightarrow F=\frac{4 \pi^{2} m}{k r^{2}}
$$

Provando, assim, que para satisfazer as Leis de Kepler, a interação gravitacional deve ser central e inversamente proporcional ao quadrado da distância.

Newton testou a validade de sua hipótese, comparando a aceleração centrípeta da Lua com a aceleração da gravidade: $\mathrm{g}=9,80 \mathrm{~m} / \mathrm{s}^{2}$.

A ideia é que a gravitação terrestre na Lua é compensada pela aceleração centrífuga do movimento circular da Lua, e esta continua em órbita por inércia e pela ausência de atmosfera no vácuo espacial, portanto, sem atrito.

Aceleração centrípeta da Lua é $a_{c}=v^{2} / r=4 \pi^{2} r / T^{2}$

Raio da órbita lunar: $r=3,84 \times 10^{8} \mathrm{~m}=384000 \mathrm{~km}$ e $T=2,36 \times 10^{6} \mathrm{~s} \cong 28 \mathrm{~d}$ 
Portanto: $a_{c}=2,72 \times 10^{-3} \mathrm{~m} / \mathrm{s}^{2}$

Logo: $g / a_{c}=9,8 /\left(2,72 \times 10^{-3}\right)=3603 \cong(60)^{2}$

Mas como o raio da Terra é: $R=6,37 \times 10^{6} \mathrm{~m}=6370 \mathrm{~km}$

Teremos: $\left(\frac{r}{R}\right)^{2}=\left(\frac{384}{6,37}\right)^{2} \simeq(60)^{2}$

Consequentemente, $g / a_{c}=(r / R)^{2}$ e, dentro da precisão dos cálculos simplificados, as duas acelerações estão na proporção inversa dos quadrados das distâncias dos pontos ao centro da Terra (ALONSO, FINN).

\subsection{ENERGIA POTENCIAL GRAVITACIONAL}

$$
\begin{gathered}
\underbrace{F_{1}=-\gamma \frac{m m^{\prime}}{r^{2}}}_{m^{\prime}} F_{2}=-F_{1}=\gamma \frac{m m^{\prime}}{r^{2}} \\
W=\int_{r_{1}}^{r_{2}} F_{2} d r=\gamma m m^{\prime}\left(\frac{1}{r_{1}}-\frac{1}{r_{2}}\right)
\end{gathered}
$$

Trabalho como variação da energia potencial entre as posições $r_{1}$ e $r_{2}$.

Trabalho realizado para afastar $m$ de $m^{\prime}$ :

Trabalho realizado é igual ao aumento da energia potencial:

$$
W=E_{p_{2}}-E_{p_{1}}=\gamma \cdot m \cdot m^{\prime}\left(\frac{1}{r_{1}}-\frac{1}{r_{2}}\right)
$$

Como referência, tomamos $r_{1}=\infty$, pois $E_{p_{1}}=0$

$$
E_{p_{2}}-0=\gamma \cdot m \cdot m^{\prime}\left(\frac{1}{\infty}-\frac{1}{r_{2}}\right) \text { A energia potencial no } \infty \text { é nula. }
$$

Logo, desprezando o índice: $E_{p}=-\gamma \frac{\mathrm{mm}^{\prime}}{r}$

Energia total de um sistema de duas partículas que interagem gravitacionalmente:

$$
E=\frac{1}{2} m v^{2}+\frac{1}{2} m^{\prime} v^{\prime 2}-\frac{\gamma \cdot m \cdot m^{\prime}}{r}, \text { isto é, } E=E_{k}+E_{p}
$$


Referindo-se ao centro de massa do sistema, a energia cinética será em função da massa reduzida $\mu$ :

$$
E_{K}=\frac{1}{2} \mu v_{12}^{2} \quad v_{12}: \text { velocidade relativa }
$$

Logo: $E=\frac{1}{2} \mu v_{12}^{2}-\frac{\gamma \cdot m \cdot m^{\prime}}{r_{12}}, \quad$ em que $\mu=\frac{m m^{\prime}}{m+m^{\prime}}=m \frac{m^{\prime}}{m+m^{\prime}}$

massa reduzida" (ver a Subseção 4.7.1 "Observações sobre massa reduzida”).

Observe a Figura a seguir, e também outro exemplo no final do Anexo 9. Consulte também Landau em Mecânica (1966) relatando ser sugestão de Newton.

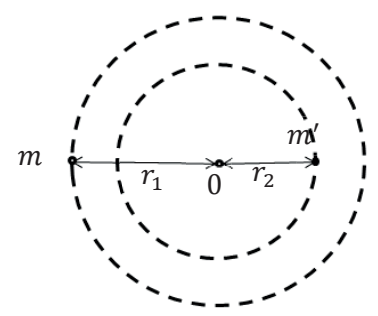

O centro de massa $r_{12}=r_{1}+r_{2}$

\subsubsection{Observações sobre massa reduzida}

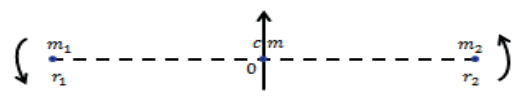

Energia cinética do conjunto $m_{1} m_{2}$. Sendo cm: centro de massa.

$$
E_{k}=\frac{m_{1} \dot{r}_{1}^{2}}{2}+\frac{m_{2} \dot{r}_{2}^{2}}{2} \text {, sendo } \dot{r}_{1}=\frac{d r_{1}}{d t}=v_{1} \text { e } \dot{r}_{2}=\frac{d r_{2}}{d t}=v_{2} \text {. }
$$

Seja $r=r_{1}-r_{2}$, distância entre os dois pontos.

Coloquemos a origem das coordenadas no centro de massa.

Teremos: $m_{1} r_{1}+m_{2} r_{2}=\left(m_{1}+m_{2}\right) r_{c m} \quad(0)$

9 Conforme a Scientific American, dividindo por $m^{\prime}: \mu=m \frac{1}{1+\frac{m}{m^{\prime}}}$, quando $m^{\prime} \gg m, \frac{1}{1+m / m^{\prime}} \rightarrow 1$, por isso na fórmula da Equação 1, da Seção 4.6 utilizamos $M$, pois $M \gg m$ e $\mu \rightarrow m$. Logo, da Equação $(1): T^{2} / a^{3}=\frac{4 \pi^{2}}{G M} \frac{1}{1+m / M}$ fazendo $m^{\prime}=M$. (4) 
Pois: $r_{c m}=\frac{m_{1} r_{1}+m_{2} r_{2}}{m_{1}+m_{2}}$, que é a média ponderada entre $r_{1}$ e $r_{2}$, tomando as massas como pesos, nesse caso $r_{c m}=0$, onde $r_{c m}$ é a distância do centro de massa.

Obtemos: $r_{1}=\frac{m_{2}}{m_{1}+m_{2}} r$

$$
\text { e } \quad r_{2}=-\frac{m_{1}}{m_{1}+m_{2}} r
$$

Colocando a origem das coordenadas respectivamente em $r_{2}$ e depois em $r_{1}$.

Substituindo em $E_{k}: E_{k}=\frac{m_{1}}{2}\left(\frac{m_{2}}{m_{1}+m_{2}}\right)^{2} \dot{r}^{2}+\frac{m_{2}}{2}\left(\frac{m_{1}}{m_{1}+m_{2}}\right)^{2} \dot{r}^{2}$

Fazendo: $\mu=\frac{m_{1} m_{2}}{m_{1}+m_{2}}$, teremos: $E_{k}=\frac{1}{2}\left[\left(\mu \frac{m_{2}+m_{1}}{m_{1}+m_{2}}\right)\right] \dot{r}^{2}=\frac{1}{2} \mu \dot{r}^{2}\left(^{*}\right)$

Chamamos $\mu$ de massa reduzida (LANDAU; ALONSO, FINN).

Quando $m^{\prime}$ for muito maior que $m\left(m^{\prime}>>m\right)$ teremos $\mu \cong m$ e $m^{\prime}$ praticamente coincide com o centro da massa [ver Seção 4.7, Equação (4)] e:

$$
E=\frac{1}{2} m v^{2}-\gamma \frac{m m^{\prime}}{r}, \text { isto é, } E=E_{k}+E_{P}
$$

Teremos três casos:

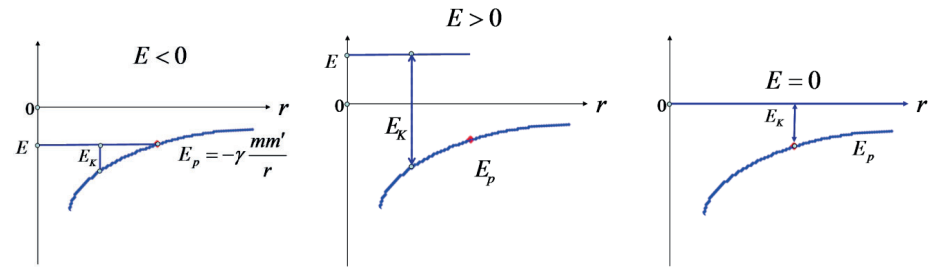

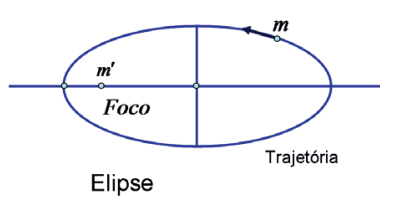

Elipse

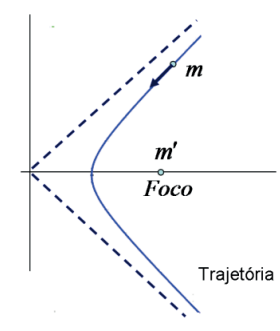

Hipérbole

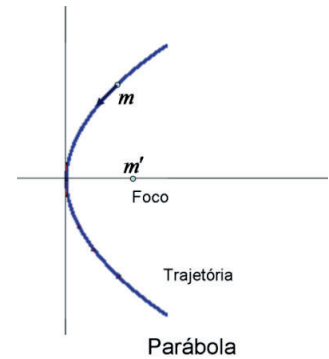

Parábola

$\left(^{*}\right) E_{K}=\frac{1}{2} \underbrace{[\frac{m_{1} m_{2}}{\left(m_{1}+m_{2}\right)} \underbrace{\frac{\left(m_{1}+m_{2}\right)}{r}}_{\left(m_{1}+m_{2}\right)}}_{\left(m_{1}+m_{2}\right)^{2}} \dot{r}^{2}=\frac{1}{2}\left[\frac{m_{2} m_{1}{ }^{2}}{\left(m_{1}+m_{2}\right)^{2}}+\frac{m_{1} m_{2}{ }^{2}}{\left(m_{1}+m_{2}\right)^{2}}\right] \dot{r}^{2}$ 
Como exemplos dessas trajetórias, podemos citar:

- Elíptica: órbitas planetárias, de cometas e satélites, excetuando as ressalvas indicadas no Anexo 9, e também as do elétron, excetuando as ressalvas indicadas pela Mecânica Quântica.

- Hiperbólica: a luz ao ser defletida quando passa próximo a uma estrela, como o Sol. Isso será mais bem visto no segundo volume desta obra.

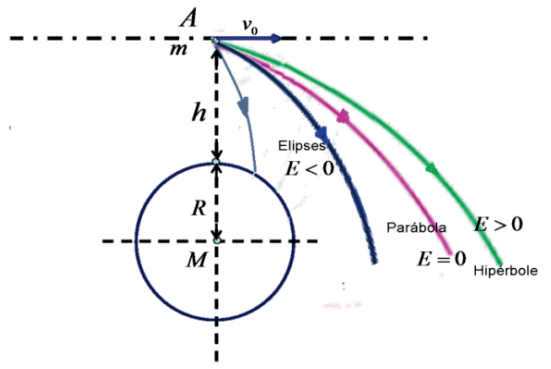

Seja um satélite a uma altura $h$ da superfície da Terra.

Energia total: $E=\frac{1}{2} m v_{0}^{2}-\gamma \frac{m M}{R+h}$

Como elipse, pode cair sobre a Terra ou orbitar em torno dela (conforme a Energia cinética). O mesmo pode ser aplicado a um satélite natural como a Lua (ALONSO, FINN).

\subsection{ENERGIA POTENCIAL GRAVITACIONAL, FUNÇÃO DAS COORDENADAS DAS PARTÍCULAS}

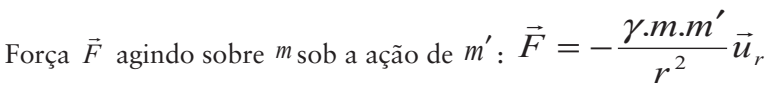

A origem do sistema está no centro de $m^{\prime}$. Como $\vec{F}$ está em direção oposta ao vetor unitário $\overrightarrow{\mathrm{u}}_{\mathrm{r}}$, seu sinal é negativo.

A força é igual ao gradiente da energia potencial com sinal trocado:

$$
\begin{gathered}
\vec{F}_{r}=-\operatorname{grad} E_{p}=-\frac{\partial E_{p}}{\partial r} \vec{u}_{r} \\
F_{r}=-\gamma \cdot m \cdot m^{\prime} /_{r^{2}} \quad \therefore \frac{\partial E_{p}}{\partial r}=\frac{\gamma \cdot m \cdot m^{\prime}}{r^{2}}
\end{gathered}
$$




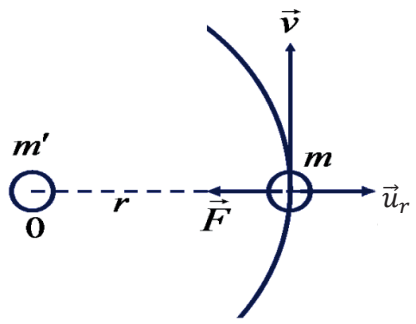

Ao integrar e dar valor 0 para energia potencial a uma distância muito grande $(r=\infty)$, obtemos:

$$
\int_{0}^{E_{p}} d E_{p}=\gamma \cdot m \cdot m^{\prime} \int_{\infty}^{r} \frac{d r}{r^{2}}
$$

Resulta em energia potencial gravitacional do sistema composto pelas massas $m$ e $m^{\prime}$ :

$$
E_{p}=-\frac{\gamma \cdot m \cdot m^{\prime}}{r}
$$

Para um sistema de mais de duas partículas interagindo gravitacionalmente, teremos:

$$
E_{p}=-\sum_{\substack{\text { Todos } \\ \text { os pares }}} \frac{\gamma \cdot m_{i} \cdot m_{j}}{r_{i j}}
$$

Conclui-se que a energia potencial é função das coordenadas das partículas, supondo um sistema de coordenadas esféricas: raio vetor $r$, ângulo com eixo $x, \phi$ e ângulo com eixo $z, \theta$ (ver a Figura a seguir; ALONSO, FINN; BORISENKO; COURANT).

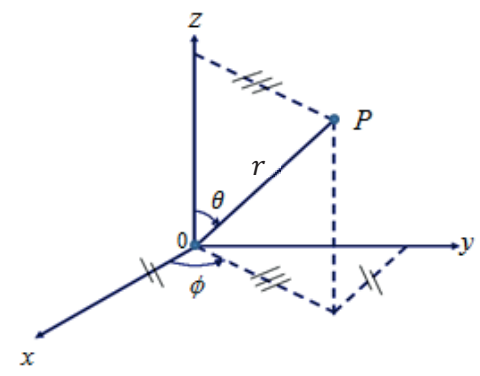

Como na Mecânica Clássica, a interação de partículas materiais é feita pela energia potencial de interação, que é função das coordenadas dessas partículas. 
Pode-se ver que essa descrição implica a instantaneidade das interações, que, aliás, era a crença de Newton. ${ }^{10}$

As forças que as outras partículas exercem sobre uma partícula dada dependem, em cada instante, nessa descrição, somente da posição dessa partícula.

A variação da posição de qualquer partícula se reflete no mesmo instante sobre as outras partículas.

Será visto no Capítulo 7 que, de acordo com a relatividade einsteiniana, o tempo não é absoluto (ver Seção 7.1) e a propagação da interação não é instantânea, mas se propaga com a velocidade da luz no vácuo (ver Seção 7.12; ALONSO, FINN; LANDAU, na Introdução, parágrafo assinalado com 1).

\footnotetext{
${ }^{10} \mathrm{Na}$ verdade, Newton não associava a interação gravitacional com a propagação da luz, como fazemos na atualidade.
} 
